\title{
A New( Natural) Way of Inhancing Labour Process: While Lying Down Supine/Left Lateral Position Meanwhile Sometimes in Between Attempt of Passing Urine in Pan/Pot By Patient Herself.
}

\author{
Dineshkumar $^{1}$, L.Ranjitsingh ${ }^{2}$, L. somen kumar $\operatorname{Singh}^{3}$, Y.Ajitkumar $\operatorname{Singh}^{4}$, \\ M.Rameswar Singh ${ }^{5}$, R.K Praneswari devi ${ }^{6}$, A. Usharani ${ }^{7}$, \\ N.Romitadevi ${ }^{8}$, Md Faridmubarak ${ }^{9}$ \\ Regional institute of medical sciences Imphal, INDIA \\ Postgraduate student, 1,9 , \\ Associate professor ${ }^{5,6}$ \\ Assistant professor ${ }^{4,7}$ \\ Senior resident ${ }^{3,8}$ \\ Professor $^{2}$
}

\begin{abstract}
.
Background: Since from many culture and long ago maternal position during labour is debated and criticized. In present era the suitable maternal position during labour and childbirth, still a matter of debate and to be standardised because, in today's standards laboring women are confined to supine-lithotomy position. ,but it results in the narrowest pelvic opening and places pressure on coccyx. while patients in case-study group were resting in bed in lying-down position as per daily practice and simultaneously instructed to pass urine in the pot near by patient,s bed irrespective of ability to pass urine or not,but attempt of passing urine was required in cases.results were compared between case and control group.

Method: This observational comparative study has been conducted at the Department of OBGY, RIMS Imphal. A total number of 140 patients were enrolled, out of which, 70 patients for the case study group and another 70 patients for control group; In control group pt were lying down on bed (supine/lateral/lithotomy position), IN study group pt were lying down on bed (supine/lateral/lithotomy position) and in between attempts of passing urine were given total 4 to 6 times and for each attempt of passing urine 4 to 6 minutes times was given .Fetomaternal outcome compared in two groups.

Result: In our study we found significant decrease in $1^{\text {st }}$ and $2^{\text {nd }}$ stage of labour in study group. There were not increase chances of instrumental delivery,caesarean section, perineal injury,PPH,manual removal of placenta or abnormal fetal distress. Rotation from Occipito post. Presentation at onset to favaroble presentation may be supportive significant .

Conclusion: Variable positions adopted by patient during labour and in between attemting to pass urine is much convenient for mothers' and more fruitful in terms of less duration of of $1^{\text {st }}$ and 2nd stage of labour,as attemting to pass urine in the pan/pot (irrespective to pass urine or not) will lead to increase gravity effect and changes in inclination of pelvis. In study we found that there were not increase chances of instrumental delivery,caesarean section, perineal injury,PPH,manual removal of placenta or abnormal fetal distress. Rotation from Occipito post. presentation at onset to favaroble presentation may be supportive significant .No significant variation is found in incidences of maternal and fetal complications.
\end{abstract}

Keywords: Labour, supine position, urine, gravity.

\section{Introduction.}

Art from many cultures long ago throughout history and anthropological studies have proved that women have preferred to give birth with their bodies vertical in sitting or squatting positions by grasping a ropes or and they sat, hung, kneeled squatted stood. Modern obstetric practice is mainly directed towards trying to make labour safe, shorter and smooth. In present era the suitable maternal position during labour and childbirth, still a matter of debate and to be standardised because, in today's standards laboring women are confined to supine-lithotomy position, for the convenience of the health personnel, but lithotomy/supine position is criticised. Modern obstetric practice is mainly directed towards trying to make labour shorter and smooth. First time the French obstetrician Mauriceau conducted delivery in bed and horizontal position was advised, for control of the childbirth process. Than it took trend in practice and the horizontal position became known as the "French Position ${ }^{(1)}$.The lithotomy side-lying or left-lateral position are the most common birth 
positions, especially in hospitals as it is one of the easiest positions to assume. Side-lying or left-lateral position it is a better option. It won't interfere with the catheter, IV line, epidural catheter or internal monitor,but it results in the narrowest pelvic opening and places pressure on coccyx. It will lead to risk of forceps or vacuum delivery and chances of episiotomy ${ }^{(2,3)}$ Lithotomy/supine positions have been criticised by many authers earlier also as ,It places pressure on blood vessels leading to the uterus and can limit blood flow to the baby. ${ }^{(4)}$ In present era we are in need of to make safe ,affordable delivery care accessible to all women irrespective to their socioeconomic,demographic and cultural level.Recent decades have seen a rapid expansion in the development and adopted many of positions designed to,accelerate ,start, augment, regulate process of labour, with the aim of improving outcomes for baby and patient. In our study during first and second stage of labour patients in control group were resting in bed in lying-down ( recumbent position ,supine or lateral) position as per modern obstetrics, while patients in case-study group were resting in bed in lying-down position as per daily practice and simultaneously instructed to pass urine in the pot/pan near by patient,s bed irrespective of ability to pass urine or not ,but attempt of passing urine was required .For passing urine patients acquired squiting, kneeling with support or standing position.

Besides resting in bed in lying-down position as per daily practice simultaneously attempts of passing urine were given total 4 to 6 times and for each attempt of passing urine 4 to 6 minutes times was given. A partogram charting and heart rate monitoring were done during the progress of labour.Oxytocin drip was administered according to uterine contractions and dose of oxytocin given was decided. Duration of first and second stage of labour was recorded. While try to pass urine in the pan during labour patient used Semisitting,sitting on pan,standing, supported squat, Walking, Leaning, squiting ,Leaning against a window, wall or bed etc.These variable positions are Comfortable. Gives good advantage of gravity to mother and baby. These positions Lengthens mother trunk and helps baby line up with the angle of patient pelvis. Movement causes changes in mother,s pelvic joints, helping baby through the birth canal it may increase patient's urge to push in the second stage of labor. In our study patient while passing urine used leaning posture, that is a nice position to use if patient want to rest but still remain upright. This position is good as engages the use of gravity. The coccyx can still move back if patient is sitting fully upright. The various positions adopted by them facilitated the process of labor. Movement helps the baby move through the pelvis, and some positions enlarge pelvic diameters.'Except for being hanged by the feet, the supine position is the worst conceivable position for labor and delivery.' Movement and positioning in labor work magic as 'Except for being hanged by the feet, the supine position is the worst conceivable position for labor and delivery.' Dr. Roberto Caldeyro-Barcia. Past president of the International Federation of Obstetricians and Gynaecologist ${ }^{(5)}$.During squating there is abduction of hip which separates the pubic bone which widens the pelvic outlet. Squatting 'straightens' the birth canal as it helps the pelvic bones to line up, rather than emphasizing the u-turn caused by the lithotomy position or the semi-sitting position. Squatting opens the pelvis by $30 \%$ more as compared to lying down position ${ }^{(6)}$ In. Urge to bear down is better in squatting position and pushing down efforts and duration is shortened. The dangling squat also helps to lengthen the body which can give the baby space to get into a better position. Inferior vena caval compression is minimal in squatting position hence foeto-placental circulation is better and chances of foetal distress is less.squatting position, gravity also plays great role in descent of the baby's head ${ }^{(7)}$

\section{Method}

This observational comparative study has been conducted at the Department of OBGY, RIMS Imphal.We conducted an observational cohort study on pregnant women admitted to the labour room of RIMS, imphal, in the Department of OBG, in the interval time between January2015and march2016.All the enrolled patients have been properly informed about the process and purpuse the study. Patients of both the groups were comparable as regards to age, parity, demographic and other parameters. All the enrolled patients have been properly informed about the aim of the study.

We considered primi parous and parity one women with uncomplicated pregnancies and single fetus in cephalic presentation at the onset of labour. According to the defined criteria, labour onset was defined by regular uterine contractions and cervical dilata- tion of atleast $3 \mathrm{~cm}$; the second labour stage was defined when a full dilatation of the cervix is attained ${ }^{(8)}$ Patients were kept under observation throughout the procedure and monitored for any discomfort and fetal heart rate.With the help of modified partograph subjects were monitored for fetal heart rate, vital signs, per vaginal examination and type, duration, and intensity of uterine contractions . A total number of 140 patients were enrolled, out of which, 70 patients for the case study group and another 70 patients for control group; were selected randomly and depending on the inclusion and exclusion criteria. Patients of both the groups were antenatally registered full term pregnancy ( 37 weeks to 40 weeks). Informed and written consent was taken from patients for including in either of the groups. They were selected randomly and depending on the inclusion and exclusion criteria.

Excluding pregnancy like.. 
1)Rh negative pregnanacy, pre-term delivery,preclampsia

2)meconium stained liquor,

3)analgesia/epidural, PROM

4)post-term pregnancy, multiple gestation, heart disease, malposition .

Patients of both the groups were comparable as regards to age, parity, demographic and other parameters. During first and second stage of labour patients in control group were resting in bed in lying-down ( recumbent position, supine or lateral) position as per modern obstetrics, while patients in case-study group were resting in bed in lying-down position as per daily practice and simultaneously instructed to pass urine in the pot near by patient's bed irrespective of ability to pass urine or not,but attempt of passing urine was required in cases(study group). (In our study focus was on attempt of passing urine.It was not necessary that pt.became able to become pass urine or not ,because in both cases and control group we evacuated bladder by intermittent catheterisation, if bladder was full). For passing urine patients acquired squiting, kneeling with support or standing position. Attempts of passing urine were given total 4 to 6 times and for each attempt of passing urine 4 to 6 minutes times in study group. A partogram charting and heart rate monitoring were done during the progress of labouR. Oxytocin drip was administered according to uterine contractions and dose of oxytocin given, was decided. Duration of first and second stage of labour was recorded. Patients were free to take any position according to their convenience. While try to pass urine in the pan during labour patient used Semisitting,sitting on pan,standing, supported squat, Walking, Leaning, squiting, Leaning against a window ,wall bed etc. Artificial rupture of membrane was done according to time and need of condition, and the progress of labor was assessed by pelvic examination after next two-hour labor was augmented by the intravenous administration of oxytocin and dose of oxytocin was adjusted according the condition of contraction.Fetal outcomes were recorded. Occurrence of birth injuries, if any, were noted down. Maternal complications were noted down for perineal injury, cervical tears, and extension of episiotomy.

III. Result

\begin{tabular}{|l|l|l|l|l|}
\hline \multicolumn{1}{|c|}{ Variables } & Cases $(\mathbf{n}=\mathbf{7 0}$ & \multicolumn{1}{c|}{ Controls $(\mathbf{n}=\mathbf{7 0}$} & \multicolumn{1}{c|}{ P value } & \multicolumn{1}{c|}{ significance } \\
\hline Maternal age in years & $24.83(3.55)$ & $24.80(4.36)$ & n.s & n.s. \\
\hline $\begin{array}{l}\text { Gestational age at } \\
\text { birth(weeks) }\end{array}$ & $39.70(1.40)$ & $39.02(1.01)$ & n.s & n.s. \\
\hline BMI & $24.24(3.50)$ & $23.28(3.54)$ & n.s & n.s. \\
\hline
\end{tabular}

\section{Study design:}

Table 1: A Comparison of study variables in two groups studied

\begin{tabular}{|l|l|l|l|l|}
\hline Variables & Cases(n=70) & Controls n=70) & P value & significance \\
\hline Episiotomy & $56(80 \%)$ & $58(82.9 \%)$ & 0.664 & n.s \\
\hline Instrumental Delivery & $8(11.4 \%)$ & $10(14.3 \%)$ & 0.614 & n.s \\
\hline $2^{\text {nd }}$ degree Perineal tear & $0(0 \%)$ & $0(0 \%)$ & 1.000 & n.s \\
\hline Caesarian section & $1(1.4 \%)$ & $5(7.1 \%)$ & 0.209 & n.s \\
\hline Perineal tear & $0(0 \%)$ & $0(0 \%)$ & 1.000 & n.s \\
\hline Abnormal FHR & $1(1.4 \%)$ & $2(2.9 \%)$ & 1.000 & n.s \\
\hline NICU Admission & $2(2.9 \%)$ & $2(2.9 \%)$ & 1.000 & n.s \\
\hline Manual removal of Placenta & $1(1.4 \%)$ & $1(1.4 \%)$ & 1.000 & n.s \\
\hline PPH & $2(2.9 \%)$ & $2(2.9 \%)$ & 1.000 & n.s \\
\hline Occipito post. Onset & $12(17.1 \%)$ & $8(11.4 \%)$ & 0.334 & n.s \\
\hline Persistent Occipito post. & $2(2.9 \%)$ & $5(7.1 \%)$ & 0.441 & n.s \\
\hline
\end{tabular}

Table 2: Comparison of $1^{\text {st }}$ stage labour an $2^{\text {nd }}$ stage labour in two groups were studied

\begin{tabular}{|l|l|l|l|l|}
\hline Variables & Cases $(\mathbf{n}=\mathbf{7 0})$ & Controls $(\mathbf{n}=\mathbf{7 0})$ & P value & Significance \\
\hline $1^{\text {st }}$ stage of labor (duration) & $180.10 \pm 118.00$ & $330.20 \pm 156.00$ & $<0.001^{* *}$ & significant \\
\hline $2^{\text {nd }}$ stage of labor (duration) & $30.20 \pm 28.00$ & $82.10 \pm 56.20$ & $<0.001^{* *}$ & significant \\
\hline
\end{tabular}

Statistical Methods: Descriptive and inferential statistical analysis has been carried out in the present study. Results on continuous measurements are presented on Mean \pm SD (Min-Max) and results on categorical measurements are presented in Number (\%). Significance is assessed at $5 \%$ level of significance.

The following assumptions on data is made,

Assumptions: 1.Dependent variables should be normally distributed, 2.Samples drawn from the population should be random, Cases of the samples should be independent.Student $t$ test ( two tailed, independent) has been used to find the significance of study parameters on continuous scale between two groups (Inter group analysis) on metric parameters. Chi-square/ Fisher Exact test has been used to find the significance of study parameters on categorical scale between two or more groups.

Significant figures

+ Suggestive significance $(\mathrm{P}$ value: $0.05<\mathrm{P}<0.10)$ 
* Moderately significant ( $\mathrm{P}$ value: $0.01<\mathrm{P} \leq 0.05$ )

** Strongly significant ( $\mathrm{P}$ value : $\mathrm{P} \leq 0.01$ ).Statistical software: The Statistical software namely SAS 9.2, SPSS 15.0, Stata 10.1, MedCalc 9.0.1 ,Systat 12.0 and R environment ver.2.11.1 were used for the analysis of the data and Microsoft word and Excel have been used to generate graphs, tables etc.

In our study we found significant decrease in $1^{\text {st }}$ and $2^{\text {nd }}$ stage of labour in study group. There were not increase chances of instrumental delivery,caesarean section, perineal injury, $\mathrm{PPH}$,manual removal of placenta or abnormal fetal distress. Rotation from Occipito post. Presentation at onset to favaroble presentation may be supportive significant.

\section{Discussion}

The ideal maternal position during labour and childbirth continues to be debated.Currently the majority of women in all cultures adopts and gives birth to their babies in non-upright positions(9)More and more women in both through out world are giving birth in health-care facilities, usually in bed in recumbent positions. A woman's position during labour has an important cultural imprint: in societies not influenced by Western culture, women progress through the first stage of labour in an upright position and change to other positions according to need(10,11,12)'Except for being hanged by the feet, the supine position is the worst conceivable position for labor and delivery.(5) In the first stage of labour vertical positions merits in association with decreased labour duration decreased pain and resulting in an increased patient's comfort ${ }^{(13,14)}$ The French obstetrician Mauriceau $(* 1637$ - $\$ 1709)$ conducted delivery in bed and horizontal position was advised, for control of the childbirth process. Than it took trend in practice and the horizontal position became known as the "French Position" (1) The "French Position" was considered convenient for health professionals; it facilitated examination and obstetric procedures for the obstetrician ${ }^{(15)}$

The American national survey "Listening to Mothers" from 2002 advised that $74 \%$ gave birth vaginally, lying on their backs throughout the second stage of labour ${ }^{16}$.In our study we found significant decrease in first and second stage of duration by instructing to pass urine and by putting effort of pass urine in the pan by study group patients. As Ganapathy et. al. ${ }^{[17]}$ Reported significant decrease in the duration of second stage of labour among women in supported sitting posture as compared to supine lithotomy position .They also reported significant difference in duration for 2 nd stage of labour. ${ }^{(17)}$ In our study we did not find any significant change in case study and control group in Episiotomy,Instrumental Delivery, $2^{\text {nd }}$ degree Perineal tear, Caesarian spectrum, Abnormal FHR, NICU Admission, Manual removal of Placenta, PPH etc.As Nasir et.al. (2007) ${ }^{(20)}$ reported no significant difference in APGAR score, abnormal FHR pattern or requirement of neo-natal resuscitation. There was no PPH in Squatting group while in Supine group 4\% of cases had retained placenta \& $1 \%$ had atonic PPH. They Reported 2\% Forceps delivery observed in Supine position. 0\% in Squatting .P. R. deJong et.al ${ }^{(19)}$ (1997) reported Fewer episiotomy . No increase in third degree tears or vulval haematoma in upright group.Allahabadia G. N. et.al. $\left(1993^{(20)}\right.$ Reported that the incidence of maternal injuries was observed in 14 cases in control group and 38 cases in squatting group.

\section{Conclusions}

Instead of lot of practicle research over many years the concept of position during labour is still debatable.Variable positions adopted by patient during labour and in between attemting to pass urine is much convenient for mothers' and more fruitful in terms of less duration of of $1^{\text {st }}$ and 2 nd stage of labour,as attemting to pass urine in the pan/pot will lead to increase gravity effect and changes in inclination of pelvis.(In our study focus was on attempt of passing urine.It was not necessary that pt.became able to pass urine or not, because in both cases and control group we evacuated bladder by intermittent catheterisation, if bladder was full).In study we found that there were not increase chances of instrumental delivery,caesarean section, perineal injury,PPH,manual removal of placenta or abnormal fetal distress. Rotation from Occipito post. Presentation at onset to favaroble presentation may be supportive significant .No significant variation is found in incidences of maternal and fetal complications.

\section{References}

[1]. Dundes, L. (1987) The evolution of maternal birthing position. Am J Public Health. 77(5): 636-41.

[2]. Nasir A, Korejo R, Noorani KJ. 2007. Child birth in squatting position. Journal of the Pakistan Medical Association. 57(1):19-22. Back.

[3]. Bodner-Adler B, Bodner K, Kimberger O, Lozanov P, Husslein P, \& Mayerhofer K. 2003. Women's position during labour: influence on maternal and neonatal outcome. Wien Klin Wochenschr. Oct 31;115(19-20):720-3. Back.

[4]. Kerr MG, Scott DB, Samuel E. 1964. Studies of the inferior vena cava in late pregnancy. British Medical Journal. 1:532-3. Back.

[5]. Caldeyro-Barcia, R. qtd in O'Mara, P, Facciolo, J, and Ponte, W. 2003. Mothering Magazine's Having a Baby, Naturally: The Mothering Magazine Guide to Pregnancy and Childbirth. Simon and Schuster. Back.

[6]. Russell JGB, Moulding of the Pelvic Outlet, J. Obsts. Gynae British Cwith, Sept. 1969, Vol. 76, 817-820 4 Russell JGB, Moulding of the Pelvic Outlet, J. Obsts. Gynae British Cwith, Sept. 1969, Vol. 76, 817-820 
[7]. Bhardwaj N, Kukade J A, Patil S, \& Bhardwaj S. Randomised controlled trial on modified squatting position of delivery. Indian Journal of Maternal and Child Health. 1995 6(2):33-39. Back.

[8]. F.G.Cunningham,K J.Leveno, S. B Bloom J.C.Hauth L.C. inWilliamsObstetrics,K.J.Leveno,Ed.,pp.409-441,McGraw- Hill,22ndedition, 2005.

[9]. Lavender, T., \& Mlay, R. (2006) Position in the second stage of labour for women without epidural anaesthesia: RHL commentary The WHO Reproduc- tive Health Library; Geneva: World Health Organization.

[10]. Andrews CM, Chrzanowski M. Maternal position, labour and comfort. Applied Nursing Research 1990;3(1):7-13

[11]. Gupta JK, Hofmeyr GJ, Smyth R. Position in the second stage of labour for women without epidural anaesthesia. Cochrane Database of Systematic Reviews 2004;Issue 1; DOI: 10.1002/14651858.CD002006.pub2.

[12]. Roberts J. Maternal position during the first stage of labour. In: Chalmers I, Enkin M, Keirse MJN eds. Effective care in pregnancy and childbirth. Vol. 2. Oxford: Oxford University Press; 1989: 883-892

[13]. E. Zwelling, “Overcoming the challenges: maternal movement andpositioningtofacilitatelaborprogress,"MCNTheAmerican JournalofMaternal/ChildNursing,vol.35,no.2,pp.72-78,2010.

[14]. S. Hunter, G. J. Hofmeyr, and R. Kulier, "Hands and knees postureinlatepregnancyorlabourforfetalmalposition(lateral or posterior)," Cochrane database of systematic reviews, no. 4, 2007.

[15]. Drife, J. (2002) The start of life: a history of obstetrics. Postgraduate Medical Journal.78: 311-315

[16]. Declercq, E., Sakala, C., Corry, M., Applebaum, S., Risher, P. (2002) Listening to mothers: Report of the first national U.S. survey of women's childbearing experiences. New York: Maternity Center Association.

[17]. Ganapathy T, Maternal birthing position and outcome of labor, The Journal of Family Welfare, Vol. 58, No. 1, June 2012, 68-73.

[18]. Ayesha Nasir, Razia Korejo, K.J.Noorani, Child Birth in Squatting position, Journal of Pakistan Medical Association, Vol. 57, No. 1, Jan 2007, pp. 19-22.

[19]. P.R.deJong, R.B.Johanson, P.Baxen, V.D.Adrians, S.vander Westhuisen, P.W.Jones, Randomised trial comparing the upright and supine positions for the second stage of labour, British Journal of Obstetrics and Gynaecology, May 1997, Vol 104, pp.567-571.

[20]. Allahbadia GN, Vaidya PR. Squatting position for delivery. J Indian Med Assoc 1993; 91: 13-16. 